EPJ Web of Conferences 38, 17012 (2012)

DOI: $10.1051 /$ epjconf/20123817012

(C) Owned by the authors, published by EDP Sciences, 2012

\title{
Quadrupole moments of odd-odd near-magic nuclei
}

\author{
D. Voitenkov ${ }^{1, a}$, O. Achakovskiy ${ }^{2}$, S. Kamerdzhiev ${ }^{1,2}$, and S. Tolokonnikov ${ }^{3}$ \\ 1 Institute for Physics and Power Engineering, 249033 Obninsk, Russia \\ 2 Insitute for Nuclear Power Engineering, National Research Nuclear University MEPhI, 249040 Obninsk, Russia \\ 3 Kurchatov Institute, 123182 Moscow, Russia
}

\begin{abstract}
Ground state quadrupole moments of odd-odd near-double-magic nuclei are calculated in the approximation of non-interacting odd neutron and odd proton. Under such a simple approximation the problem is reduced to the calculations of quadrupole moments of corresponding odd-even nuclei. These calculations are performed within the self-consistent Theory of Finite Fermi Systems based on the Energy Density Functional by Fayans et al. with the known DF3-a parameters. A reasonable agreement with the available experimental data is obtained for odd-odd nuclei and odd near-magic nuclei investigated. The self-consistent approach under consideration allowed us to predict the unknown quadrupole moments of odd-even and odd-odd nuclei near the double-magic ${ }^{56,78} \mathrm{Ni},{ }^{100,132} \mathrm{Sn}$ nuclides.
\end{abstract}

\section{Introduction}

One of the challenging goals of modern nuclear theory is to elaborate the approaches which would enable to predict the characteristics of unstable nuclei. Therefore, a developed theoretical approach should have a very high predictive power. The Energy Density Functional (EDF) approach is one of such approaches. As a rule, odd-odd nuclei are unstable ones, so here we will consider their characteristics using the self-consistent Theory of Finite Fermi Systems (TFFS) [1] based on the EDF by Fayans et al. [2].

Quite recently, a good description of the ground state quadrupole [3,4] and magnetic[5] moments of odd nearand semi-magic nuclei has been obtained within this selfconsistent approach where the "single quasi-particle approximation" developed in the framework of the standard TFFS [6] was used. This approximation means that one quasi-particle with the local charge $e_{q}$ is added to the eveneven core and the core is polarized due to the LandauMigdal (LM) interaction between the particle considered and the core nucleons. In other words, the quasi-particle possesses the effective charge $e_{\text {eff }}$ caused by the polarizability of the core, which should be found by solving the TFFS equations. Within the TFFS, static moments of odd nucleus are determined by the diagonal matrix element of the effective field $V$ in the external field $V_{0}$ (see Eq. (6) below).

As odd-odd nuclei are more complicated objects than the even-odd ones, here we only consider the odd-odd nearmagic nuclei together with the corresponding odd nearmagic ones. Within the above-mentioned self-consistent EDF approach, we calculate the ground state quadrupole moments of odd-odd near-magic nuclei supposing for simplicity, that direct interaction between odd neutron and odd proton vanishes.

As we will see, such a simple approximation allows us to check this approximation rather successfully in a pure phenomenological way.

\footnotetext{
a e-mail: voitenkov@list.ru
}

\section{Quadrupole moments of odd and odd-odd near-magic nuclei}

Within the TFFS [6], the static quadrupole moment $Q_{\lambda}$ of an odd near-magic nucleus with the odd nucleon in the state $\lambda \equiv(n, j, l, m)$ can be found in terms of the diagonal matrix element of the effective field $V$ :

$$
Q_{\lambda}=\langle\lambda|V(\omega=0)| \lambda\rangle=c_{j}\langle n j l\|V\| n j l\rangle,
$$

where $c_{j}=2 j(2 j-1)^{1 / 2}[(2 j+3)(2 j+2)(2 j+1) 2 j]^{-1 / 2}$. In the framework of the above-mentioned TFFS single quasiparticle approximation and of our main approximation that there is no interaction between two quasi-particles, the quadrupole moment of an odd-odd nucleus with spin $I$ is as follows:

$$
Q_{I}=<I I\left|V^{p}+V^{n}\right| I I>
$$

where $\Psi_{I I}=\Sigma \varphi_{1} \varphi_{2}<j_{1} m_{1} j_{2} m_{2} \mid I I>$ for the case of odd particle-odd particle. Here, $\varphi_{1}$ is the single-particle wave function with the quantum numbers $1 \equiv \lambda_{1} \equiv\left(n_{1}, j_{1}\right.$, $\left.l_{1}, m_{1}\right)$. In the limit of no interaction between the core and odd quasi-particles the effective fields $V^{p}$ and $V^{n}$ should be replaced by $e_{q}^{p} V_{0}$ and $e_{q}^{n} V_{0}$, see Eq. (6) below.

Then the expression for ground state quadrupole moments of odd-odd near-magic nuclei has the form (for the case of particle-particle or hole-hole):

$$
\begin{gathered}
Q_{I}=(2 I+1)\left(\begin{array}{ccc}
I & 2 & I \\
I & 0 & -I
\end{array}\right)(-1)^{j_{p}+j_{n}+I+2} \times \\
\times\left[\left\{\begin{array}{ccc}
j_{p} & I & j_{n} \\
I & j_{p} & 2
\end{array}\right\} c_{j_{p}}^{-1} Q^{p}+\left\{\begin{array}{ccc}
j_{n} & I & j_{p} \\
I & j_{n} & 2
\end{array}\right\} c_{j_{n}}^{-1} Q^{n}\right],
\end{gathered}
$$

and for the case of particle-hole we have

$$
\begin{array}{r}
Q_{I}=(2 I+1)\left(\begin{array}{ccc}
I & 2 & I \\
I & 0 & -I
\end{array}\right)(-1)^{j_{p}+j_{n}+I+2} \times \\
\times\left[\left\{\begin{array}{ccc}
j_{p} & I & j_{n} \\
I & j_{p} & 2
\end{array}\right\} c_{j_{p}}^{-1} Q^{p}+(-1)^{j_{p}-j_{n}}\left\{\begin{array}{ccc}
j_{n} & I & j_{p} \\
I & j_{n} & 2
\end{array}\right\} c_{j_{n}}^{-1} Q^{n}\right],(4)
\end{array}
$$


where $Q^{p}$ and $Q^{n}$ are the quadrupole moments of corresponding odd nuclei which are determined by Eq.(1). A similar formula can be easily obtained for the hole-particle case.

Thus the problem is reduced to the calculations of quadrupole moments of corresponding odd-even nuclei. One can take the values of quadrupole moments of odd nuclei from the experiment (phenomenological approach, Sec. 3) and one can also calculate them and obtain the quadrupole moments of corresponding odd-odd nuclei according to Eqs. (3), (4) (microscopic approach, Sec. 4)

\section{Phenomenological approach}

We found only three odd-odd nuclei where there are all three necessary experimental values. Our main approximation is confirmed by a reasonable agreement with the experiment. This is demonstrated in Table 1, where the experimental values of quadrupole moments of corresponding odd nuclei are used. The experimental data used here and in what follows are taken from [7].

Table 1. Quadrupole moments $Q$ (in e.b) of odd-odd near-magic nuclei (phenomenological approach).

\begin{tabular}{lcccc}
\hline \hline nucl. & $J^{\pi}$ & $T_{1 / 2}$ & $Q_{\text {phen }}$ & $Q_{\exp }$ \\
\hline${ }_{19}^{40} \mathrm{~K}_{21}$ & $4^{-}$ & $1.248 \times 10^{9} \mathrm{y}$ & $-0.106(6)$ & $-0.071(1)$ \\
${ }_{92}^{92} \mathrm{Nb}_{51}$ & $7^{+}$ & $3.47 \times 10^{7} \mathrm{y}$ & $-0.43(7)$ & $-0.35(3)$ \\
${ }_{21}^{210} \mathrm{Bi}_{127}$ & $1^{-}$ & $5.01 \mathrm{~d}$ & $+0.22(6)$ & $+0.19(6)$ \\
& & & & \\
\hline \hline
\end{tabular}

More convincing results which confirm our approximation were obtained for the static ground state magnetic moments of odd-odd near-magic nuclei with much wider set of experimental data (see Ref. [8]).

\section{Self-consistent calculations}

These calculations are performed within the self-consistent Theory of Finite Fermi Systems (TFFS) based on the Energy Density Functional (EDF) by Fayans et al. with the DF3-a parameters [9]. The details of the calculations for odd nuclei were described in Ref. [3]. Here we summarize several formulas which are required to understand the main ingredients of the approach. In this method, the ground state energy of a nucleus is considered as a functional of normal and anomalous densities,

$$
E_{0}=\int \mathcal{E}\left[\rho_{n}(\mathbf{r}), \rho_{p}(\mathbf{r}), v_{n}(\mathbf{r}), v_{p}(\mathbf{r})\right] d^{3} r
$$

According to Eq.(1), the static quadrupole moment $Q_{\lambda}$ of an odd nucleus when the odd nucleon is in the state $\lambda$ can be found by solving the equation for the effective field $V$ in the static external field $V_{0}(\mathbf{r})=\sqrt{\frac{16 \pi}{5}} r^{2} Y_{20}(\mathbf{n})$.

$$
\hat{V}(\omega)=\hat{e}_{q} V_{0}(\omega)+\hat{\mathcal{F}} \hat{A}(\omega) \hat{V}(\omega)
$$

where $e_{q}$ is the local quasi-particle charge with respect to the external field $V_{0}$ and all terms are matrices in the isotopic space. In the standard TFFS notations, the particlehole propagator $A$ in the coordinate representation reads

$$
\begin{array}{r}
A\left(\mathbf{r}, \mathbf{r}^{\prime}, \omega\right)=-\sum_{1} n_{1} \varphi_{1}^{*}(\mathbf{r}) \varphi_{1}\left(\mathbf{r}^{\prime}\right) \times \\
\times\left[G\left(\mathbf{r}^{\prime}, \mathbf{r} ; \epsilon_{1}+\omega\right)+G\left(\mathbf{r}^{\prime}, \mathbf{r} ; \epsilon_{1}-\omega\right)\right]
\end{array}
$$

where $n_{1}=(0,1)$ are occupation numbers, summation is over states below the Fermi surface and the known singleparticle Green functions $G$ already contain the entire singleparticle continuum. Just due to this feature of the Green function, the single-particle continuum is taken into account completely in the TFFS.

In our case, the local charges in Eq. (6) are $e_{q}^{p}=1, e_{q}^{n}=$ 0 . The explicit form of the above equations is written down for the case of the electric ( $t$-even) symmetry we deal with. In Eq. (6), $\mathcal{F}$ is the usual LM amplitude,

$$
\mathcal{F}=\frac{\delta^{2} \mathcal{E}}{\delta \rho^{2}},
$$

Using formulas (5) - (8) we have calculated self-consistently ground state quadrupole moments of odd and corresponding odd-odd near-magic nuclei. The method used has a high predictive power and, according to Eq. (7), takes into account completely the single-particle continuum, which is especially important for nuclei with a small separation energy, like ${ }^{78} \mathrm{Ni}\left(S_{n} \approx 5.8 \mathrm{MeV}\right)$ and ${ }^{100} \mathrm{Sn}\left(S_{p} \approx 2.4\right.$ $\mathrm{MeV})$. For this reason, first of all we have calculated the quadrupole moments of the odd and odd-odd nuclei near unstable double-magic nuclei ${ }^{56} \mathrm{Ni},{ }^{78} \mathrm{Ni},{ }^{100} \mathrm{Sn}$ and ${ }^{132} \mathrm{Sn}$.

\subsection{Odd near-magic nuclei}

The final expression for the quadrupole moment of an odd nucleus, Eq.(1), is as follows:

$$
Q_{\lambda}=V_{\lambda}= \pm \frac{2 j-1}{2 j+2} \int V(r) R_{n l j}^{2}(r) r^{2} d r
$$

where the (-) sign should be taken for the odd particle and $(+)$ stands for the odd hole [10]. (The replacement of the effective field $V$ by $e_{q} V_{0}$ which was considered in [10] does not change the sign). The $j$-dependent factor in Eq.(9) appears due to the angular integral. For $j>1 / 2$ it is always negative. The equation for $V(r)$, which was obtained from Eq.(6), is solved in the coordinate space using Eqs.(7)-(8). The same set of DF3-a parameters was used to calculate the self-consistent single-particle basis and the effective LM interaction.

The results of calculations are shown in Table 2 and Table 3, where in column $Q_{\text {theor }}$ the self-consistent values are shown for odd nuclei near double-magic nuclei ${ }^{56,78} \mathrm{Ni},{ }^{100,132} \mathrm{Sn},{ }^{208} \mathrm{~Pb}$. To compare with the well-known phenomenological description, the results with the effective charger $e_{e f f}^{p}=2, e_{e f f}^{n}=1$ are represented in columns $Q_{\text {eff }}$, where they have been calculated with the same selfconsistent single-particle basis. These values have been justified microscopically within TFFS in Ref. [11] where they have been introduced as $\left(e_{e f f}^{p}\right)_{\lambda}=V_{\lambda}^{p} /\left(V_{0}\right)_{\lambda}$ and $e_{e f f}^{n}=$ $V_{\lambda}^{n} /\left(V_{0}\right)_{\lambda}$. Note that in nuclei with paring these values are 
$e_{e f f} \cong 4-6$ [3] due to the contribution of unfilled nuclear shells. Comparing the values in columns $Q_{\text {eff }}$ and $Q_{\text {theor }}$, one can see that the use of the phenomenological effective charges $e_{e f f}^{p}=2, e_{e f f}^{n}=1$ is not always good quantitatively.

Table 2. Quadrupole moments $Q$ (e-b) of odd-neutron near-magic nuclei.

\begin{tabular}{lccccc}
\hline \hline Nucleus & $J^{\pi}$ & $T_{1 / 2}$ & $Q_{\text {eff }}$ & $Q_{\text {theor }}$ & $Q_{\exp }$ \\
\hline${ }_{55}^{55} \mathrm{Ni}_{27}$ & $7 / 2^{+}$ & $204.7 \mathrm{~ms}$ & 0.11 & 0.26 & - \\
${ }^{57} \mathrm{Ni}_{29}$ & $3 / 2^{-}$ & $35.6 \mathrm{~h}$ & -0.07 & -0.17 & - \\
${ }_{28}^{77} \mathrm{Ni}_{49}$ & $(9 / 2)^{+}$ & $128 \mathrm{~ms}$ & 0.16 & 0.20 & - \\
${ }_{28}^{79} \mathrm{Ni}_{50}$ & $\left(5 / 2^{+}\right)$ & $635 \mathrm{~ns}$ & -0.17 & -0.12 & - \\
${ }_{29}^{101} \mathrm{Sn}_{51}$ & $(5 / 2)^{+}$ & $1.7 \mathrm{~s}$ & -0.13 & -0.21 & - \\
${ }_{50}^{13} \mathrm{Sn}_{81}$ & $\left(3 / 2^{+}\right)$ & $56 \mathrm{~s}$ & 0.10 & 0.10 & $-0.04(8)$ \\
${ }_{50}^{13} \mathrm{Sn}_{83}$ & $7 / 2^{-}$ & $1.46 \mathrm{~s}$ & -0.23 & -0.17 & - \\
${ }_{50}^{207} \mathrm{~Pb}_{125}$ & $(1 / 2)^{-}$ & stable & 0 & 0 & - \\
${ }_{82}^{209} \mathrm{~Pb}_{127}$ & $(9 / 2)^{+}$ & $3.253 \mathrm{~h}$ & -0.29 & -0.26 & $-0.3(2)$ \\
${ }_{82} \mathrm{~Pb}_{127}$ & & & & & \\
\hline \hline
\end{tabular}

\subsection{Odd-odd near-magic nuclei}

Using Eqs.(3),(4) and the values of quadrupole moments of odd nuclei presented in Tables 2 and 3, we have calculated quadrupole moments of corresponding odd-odd nuclei with both the phenomenological values $e_{\text {eff }}^{p}=2, e_{\text {eff }}^{n}=$ 1 (column $Q_{\text {eff }}$ ) and the self-consistently calculated quadrupole moments of odd nuclei (column $Q_{\text {theor }}$ ), see Table 4. Unfortunately, the experimental data are scarce so the $Q_{\text {theor }}$ values in Table 4 are our predictions of the quadrupole moments in the exotic unstable nuclei under consideration.

\section{Conclusion}

A reasonable agreement with all available experimental data for quadrupole moments in odd and odd-odd nearmagic nuclei has been obtained in our self-consistent calculations using the approximation of no interaction between odd particles.

The self-consistent approach has a high predictive power required to describe properties of nuclei where there is no experimental data. In this connection, our predictions for quadrupole moments of nuclei near unstable ${ }^{56,78} \mathrm{Ni}$, ${ }^{100,132} \mathrm{Sn}$ are of special interest.

\section{Acknowledgements}

The authors thank E.E. Saperstein and V.I. Isakov for useful discussions. The work was partly supported by the DFG and RFBR Grants Nos.436RUS113/994/0-1 and 09-02-91352NNIO-a, by the Grants NSh-7235.2010.2 and 2.1.1/4540 of the Russian Ministry for Science and Education, and by the RFBR grants 11-02-00467-a and 12-0200955-a.
Table 3. Quadrupole moments $Q$ (e-b) of odd-proton near-magic nuclei.

\begin{tabular}{|c|c|c|c|c|c|}
\hline Nucleus & $J^{\pi}$ & $T_{1 / 2}$ & $Q_{\text {eff }}$ & $Q_{\text {theor }}$ & $Q_{\text {exp }}$ \\
\hline${ }_{27}^{55} \mathrm{Co}_{28}$ & $7 / 2^{-}$ & $17.53 \mathrm{~h}$ & 0.22 & 0.31 & - \\
\hline${ }_{29}^{77} \mathrm{Cu}_{28}$ & $3 / 2^{-}$ & $196.3 \mathrm{~ms}$ & -0.15 & -0.20 & - \\
\hline${ }_{29}^{79} \mathrm{Cu}_{50}$ & $\left(3 / 2^{-}\right)$ & $188 \mathrm{~ms}$ & -0.14 & -0.13 & - \\
\hline${ }_{49}^{99} \operatorname{In}_{50}$ & $(9 / 2)^{+}$ & $3 \mathrm{~s}$ & 0.35 & 0.35 & - \\
\hline${ }_{49}^{431} \mathrm{In}_{82}$ & $(9 / 2)^{+}$ & $0.28 \mathrm{~s}$ & 0.40 & 0.28 & - \\
\hline${ }_{51}^{133} \mathrm{Sb}_{82}$ & $(7 / 2)^{+}$ & $2.34 \mathrm{~m}$ & -0.34 & -0.23 & - \\
\hline${ }_{81}^{207} \mathrm{Tl}_{126}$ & $1 / 2^{+}$ & $4.77 \mathrm{~m}$ & 0 & 0 & - \\
\hline${ }_{83}^{209} \mathrm{Bi}_{126}$ & $(9 / 2)^{-}$ & stable & -0.51 & -0.34 & $\begin{array}{l}-0.37(3), \\
-0.55(1), \\
-0.77(1), \\
-0.40(5)\end{array}$ \\
\hline
\end{tabular}

Table 4. Quadrupole moments $Q$ (e.b) of odd-odd near-magic nuclei.

\begin{tabular}{lccccc}
\hline \hline Nucleus & $J^{\pi}$ & $T_{1 / 2}$ & $Q_{\text {eff }}$ & $Q_{\text {theor }}$ & $Q_{\text {exp }}$ \\
\hline${ }_{27} \mathrm{Co}_{27}$ & $0^{+}$ & $193.28 \mathrm{~ms}$ & - & - & - \\
${ }_{56} \mathrm{Co}_{29}$ & $4^{+}$ & $77.236 \mathrm{~d}$ & 0.19 & 0.30 & $+0.25(9)$ \\
${ }_{56}^{26} \mathrm{Cu}_{27}$ & $\left(4^{+}\right)$ & $93 \mathrm{~ms}$ & 0.14 & 0.28 & - \\
${ }_{29}^{28} \mathrm{Cu}_{29}$ & $1^{+}$ & $3.204 \mathrm{~s}$ & 0.09 & 0.15 & - \\
${ }_{79}^{78} \mathrm{Cu}_{49}$ & $\left(3^{-}\right)$ & $637 \mathrm{~s}$ & -0.18 & -0.21 & - \\
${ }_{29}$ & $\left(4^{-}\right)$ & & $4 \times 10^{-5}$ & -0.03 & - \\
& & 0.24 & 0.21 & - \\
${ }_{49}^{100} \mathrm{In}_{51}$ & $\left(6^{+}\right)$ & $5.9 \mathrm{~s}$ & 0.21 & - \\
${ }_{130} \mathrm{In}_{81}$ & $1^{-}$ & $0.29 \mathrm{~s}$ & -0.08 & -0.07 & - \\
${ }_{132} \mathrm{In}_{83}$ & $\left(7^{-}\right)$ & $0.207 \mathrm{~s}$ & -0.40 & -0.29 & - \\
${ }_{49}^{132} \mathrm{Sb}_{81}$ & $(4)^{+}$ & $2.79 \mathrm{~m}$ & -0.30 & -0.22 & - \\
${ }_{51}^{134} \mathrm{Sb}_{83}$ & $\left(0^{-}\right)$ & $0.78 \mathrm{~s}$ & - & - & - \\
${ }_{51}^{206} \mathrm{Tl}_{125}$ & $0^{-}$ & - & - & - & - \\
${ }_{81}^{208} \mathrm{Tl}_{127}$ & $5^{+}$ & $3.053 \mathrm{~m}$ & -0.30 & -0.27 & - \\
${ }_{81}^{208} \mathrm{Bi}_{125}$ & $5^{+}$ & $3.68 \mathrm{E}+5 \mathrm{y}$ & -0.51 & -0.35 & $-0.64(6)$ \\
${ }_{83}^{210} \mathrm{Bi}_{127}$ & $1^{-}$ & $5.012 \mathrm{~d}$ & 0.21 & 0.16 & $+0.136(1)$ \\
${ }_{83}$ & & & & & \\
\hline \hline
\end{tabular}

\section{References}

1. V. Khodel, E.E. Saperstein, Phys. Rep. 92, 183 (1982)

2. S.A. Fayans, S.V. Tolokonnikov, E.L. Trykov, and D. Zawischa, Nucl. Phys. A 676, 49 (2000)

3. S.V. Tolokonnikov, S. Kamerdzhiev, D. Voitenkov, S. Krewald, and E.E. Saperstein, arXiv:1107.2432v2[nuclth]; Phys. Rev. C 84, 064324 (2011)

4. S.V. Tolokonnikov, S. Kamerdzhiev, D. Voitenkov, S. Krewald, and E.E. Saperstein, EPJA 48:70 (2012)

5. I.N. Borzov, S.V. Tolokonnikov and E.E. Saperstein, Phys. Atom. Nucl. 71, 469 (2008)

6. A.B. Migdal, Theory of finite Fermi systems and applications to atomic nuclei (Wiley, New York, 1967)

7. N. Stone, At. Data Nucl. Data Tables 90, 75 (2005)

8. S.P.Kamerdzhiev, O.I. Achakovskiy, D.A. Voitenkov and S.V. Tolokonnikov, Phys. At. Nucl., to be published.

9. S.V. Tolokonnikov, E.E. Saperstein, Phys. At. Nucl. 73, 1684 (2010)

10. A. Bohr and B.R. Mottelson, Nuclear Structure (Benjamin, New York, Amsterdam, 1969.), Vol. 1.

11. S.P.Kamerdzhiev, Sov. J. Nucl. Phys. 2, 415 (1965) 